\title{
Characterization of The Microbiome That Colonizes The Digestive Tract of Calves From 0 To 42 Days of Age, Raised In The Mexican Tropics.
}

Carolina Robles Rodríguez

Posgrado en Ciencias de la Producción y de la Salud Animal, UNAM

María Laura González Dávalos

UNAM: Universidad Nacional Autonoma de Mexico

Armando Shimada

UNAM: Universidad Nacional Autonoma de Mexico

Carla Daniela Robles Espinoza

UNAM: Universidad Nacional Autonoma de Mexico

Maria Ofelia Mora ( $\nabla$ ofemora66@unam.mx )

UNAM: Universidad Nacional Autonoma de Mexico https://orcid.org/0000-0002-7073-7740

\section{Research Article}

Keywords: Calves, Microbial communities, Metagenomic, Rumen, Jejunum

Posted Date: July 28th, 2021

DOl: https://doi.org/10.21203/rs.3.rs-741679/v1

License: (9) This work is licensed under a Creative Commons Attribution 4.0 International License. Read Full License 


\section{Abstract}

In the first few weeks of a calf's life, the early colonization of microbes throughout the gastrointestinal tract (GIT) is critical in its digestive system and immunity development. Analyses of data generated from next-generation sequencing platforms have revolutionized the understanding of host-associated microbial communities; these analyses can be done through a variety of bioinformatics pipelines. This study aimed to describe the diversity and evolution of gastrointestinal tract microbial communities in the first days of life of calves; for which we used the USEARCH and KRAKEN2 algorithms, in terms of alpha and beta diversity analysis in the rumen and jejunum contents of calves from $0,7,28,42$ days-born in the Mexican Tropic and its changes. The two pipelines detected similar abundance distributions in the three most abundant taxa Proteobacteria, Firmicutes, and Bacteroidetes. The present study revealed the changes occurring in the rumen and jejunum ecosystem in the first week of life, reflected by a decline in the phylum Proteobacteria and an increase in phylum Bacteroidetes in rumen and Firmicutes in the jejunum. We observed an increase in the similarity in the phylum taxa with age, suggesting a homogeneous and specific mature community, compared with the primary community.

\section{Introduction}

Ruminants and the microorganisms in their digestive tract have evolved to create a symbiosis that has allowed them to adapt their diet to the consumption of forages, obtaining the necessary energy for all metabolic processes. Relationships between digestive tract bacterial communities and their mammalian hosts have been shown to have an important role in the host's well-being and proper function.

Colonization by the initial microbiota is influenced by many factors such as diet, feeding methods (breastfeeding or artificial rearing), birth canal microbiota, and the environment where the animal grows up. This is a dynamic, stable process, but changes in the proportions of microorganism taxa are dependent on the surrounding metacommunity, which is important to achieve a correct function that can influence the health, immunity, and productivity of animals when reaching adulthood (Jiao et al. 2005).

The microbiome of the digestive tract of ruminants was first investigated using bacterial culture techniques, however, with this methodology, it was only possible to identify $11 \%$ of the species (Pitta et al. 2009) since it was not possible to grow a large proportion of bacteria in laboratory conditions. The emergence of the new generation of sequencing techniques has allowed us to expand our knowledge about the bacterial species of the digestive tract and to understand the interactions between the host and the microbiota, and their impact on the development of immunity and health. In this study, we set out to study the changes in the microbiota of calves of the Mexican Tropic, to dissect the possible microbial colonization pattern in the gastrointestinal tract of calves.

\section{Materials And Methods}

\section{Animals and sampling}


The experimental protocols (CICUAE.DC-2019/4 - 2) were approved by the internal committee for the experimental animal care and use (CICUAE, UNAM) according to the NOM-062-ZOO-1999. The animals were obtained from the Centro de Enseñanza, Investigación y Extensión en Ganadería Tropical (CEIEGT), Tlapacoyan, Veracruz.

A total of 12 newborn calves $(n=3)$ of ages $0,7,28$, and 42 days were used. The three animals of 0 days were sacrificed immediately after birth, without having ingested colostrum (Zhong et al. 2017). The calves of ages 7,28 , and 42 days had the same management in the livestock unit (up to sacrifice), which consists of colostrum ingestion and staying with the dam for 4 days in a maternity pen. After this period, the calves were separated from the cow and moved to the rearing area with the rest of the calves where they were fed a milk substitute ( $22 \%$ protein and $12 \%$ fat), roughage ad libitum, and from the second week, a concentrate.

Ruminal and intestinal (jejunum) contents were collected from all animals. The samples were immediately frozen in liquid nitrogen for further analysis (Liang et al. 2014 and 2016).

\section{DNA extraction}

To determine bacterial diversity, genomic DNA was extracted from samples previously collected. DNA extraction was performed according to the RBB $+C$ method (Yu and Morrison 2004); zirconium beads and lysis buffer were used for cell disruption. 10M ammonium acetate, isopropanol, and $70 \%$ ethanol were used for the precipitation of nucleic acids. Finally, for protein removal and DNA purification, the columns of the QIAamp DNA Mini Kit (QIAGEN, Hilden, Germany) were used.

DNA concentration was quantified using a Qubit 3.0 fluorometer (Life Technologies, Carlsbad, CA, USA). Product integrity was observed on $1 \%$ agarose gel. Subsequently, the samples were sent to RTL genomics to perform the sequencing of the DNA samples, using primers covering the V4 region (357wF:3'CCTACGGGNGGCWGCAG-5'; 785R:3'-GACTACHVGGGTATCTAATCC-5'). The Illumina MiSeq platform was used with paired readings $(2 \times 250)$.

Data quality control and analyses were performed using the USEARCH V.11 pipeline. The quality control was applied to the fastq files, which included removing adapters and cutting the sequences to lengthbased filtering of $400 \mathrm{bp}$ ( $<200 \mathrm{bp}$ was excluded from the analysis). The next step was to align the obtained sequences to define operational taxonomic units (OTUs) for eventual taxonomy assignment; the OTUs table was generated at $97 \%$ identity. The UCLUST method was used to cluster the reads into OTUs (Edgar, 2010).

For KRAKEN2 (Wood et al. 2019), fastq files were firstly uploaded into FastQC for quality control and removal of ambiguous sequences, and then the software Trimmomatic (version 0.32; Bolger et al. 2014) was used to trim residual artificial sequences, cut bases with quality scores below 20 , and filtering of 400 bp ( $<200$ bp was excluded from the analysis). After pre-processing, the protocol proposed by Lu et al. (2020) was used. 
Taxonomy was assigned using RDP V.16 against the $16 \mathrm{~S}$ reference database with $97 \%$ identity for both pipelines (Edgar, 2010). The data were analyzed on the R platform with the help of the pyloseq (McMurdie et al. 2013) library. Alpha diversity values for bacterial communities with ruminal and jejunum content were obtained using various diversity indexes (observed OTUs, Chao's estimate, Shannon's diversity index).

\section{Statistical analysis}

Additional statistical analyses were performed using a completely random model using the GLM function of the SAS statistical program (SAS 9.3):

$y_{j k}=\mu+D_{j}+\varepsilon_{(j) k}$

$Y_{j k}=$ response variable of $j^{\text {th }}$ day

$\mu=$ general mean

$D_{j}=$ effect of day $j$

$\nabla_{(j) k}=$ associated error of the $k^{\text {th }}$ sample of the $j^{\text {th }}$ day.

\section{Results}

\section{Rumen bacterial composition across different age groups}

Alpha diversity metrics summarize the structure of an ecological community concerning its richness (number of taxonomic groups), uniformity (distribution of group abundances), or both.

Alpha-diversity was measured using Shannon, Chao estimate, and observed OTUs (Fig. 1). Observed OTUs were greater in the 28-day sample in the rumen $(418.3 \pm 42.19)$ compared to the other days in both samples rumen and jejunum. Concerning metrics related to the richness, the Chao estimate for rumen samples increased from 0-day to 42-day.; samples of day 28 having the greater richness following by 42 day samples $(476.23 \pm 52.23 ; 368.60 \pm 52.23$ respectively; $P<0.05)$, we did not find a difference in the jejunum $(P>0.05)$. Diversity did not fluctuate greatly $(P>0.05)$ and was similar for all the trials in both regions (rumen and jejunum).

Analyzing the abundance of the regions, we found that the Firmicutes, Proteobacteria, Bacteroidetes, and Actinobacteria phyla represent almost $90 \%$ of all species found in both rumen and jejunum.

In the case of the rumen (Fig. 2), a greater proportion of the Proteobacteria phylum was observed on day 0 (decreasing from $34 \%$ on 0 -day to $18.7 \%$ on 42 -day), which was replaced by an increasing proportion of the Bacteroidetes phylum which remains as the more abundant phylum throughout all later measurements (increasing from $16.6 \%$ on 0 -day to $70 \%$ on 42 -day; $P \leq 0.05$, Fig. 2). Firmicutes and 
Actinobacteria phyla on day 0 represent $24.7 \%$ and $13.7 \%$, respectively of the abundance in the rumen ( $P$ $\leq 0.05$ ), this diminished on days 7 and 28 , by day 42 it represents $20 \%$ for Firmicutes and $8 \%$ for Actinobacteria.

These findings were supported by both programs, with small differences found between them. KRAKEN2 detected an increase in the proportion of the phylum Firmicutes on day 0 (decreasing from $45-18 \%$ by day $42 P \leq 0.05$; similar to USEARCH), and the two programs agree on the proportions of the phylum on days $7,28,42$.

Jejunum samples were dominated by Firmicutes phylum in older ages, being the most abundant phylum in 7,28 , and 42 -days samples, representing $39 \% ; 87 \% ; 74 \%$ of the abundance respectively, according to both programs (Fig. 3). On the other hand, there is a decrease in the age of the Proteobacteria phylum (decreasing from $70 \%$ on 0 -day to $5 \%$ on 42 -day), similar to what is observed in the rumen. Actinobacteria phylum did not show a difference between the days under study $(P>0.05)$. Bacteroidetes phylum has an increase in 7 -day representing $24 \%$ of the abundance for this day and a decrease on day 42 representing less than $10 \%$ of the phylum for this day.

We also analyzed the microbiome shared in each region (microbial core) among the four ages, the jejunum microbiota had the most (OTUs) in common with and those of the rumen sample had the least.

This similarity between the newborn and older calves microbiota was evident when comparing the jejunum sample's microbial composition (Fig. 4). As a group, newborns shared more phylum-level taxa with older ages (114 of OTUs were shared by all jejunum samples in USEARCH analysis (Fig. 4b), and 107 of OTUs were shared by all jejunum samples in KRAKEN2 analyses (Fig. 4d). In rumen only, 11 of the OTUs analyzed with USEARCH were exclusively shared between all ages, and only 8 of the OTUs analyzed with KRAKEN2 (Fig. 4a and 4c) were shared by all ages; rumen 0-day had the greatest amount of individual OTUs (204 OTUs detected with USEARCH, and 355 OTUs detected with KRAKEN2 were not shared with other ages).

\section{Discussion}

A diverse microbial population colonizes the sterile mammalian gastrointestinal tract during and after birth. There is increasing evidence that this complex microbiome plays a crucial role in the development of the mucosal immune system and influences newborn health. Microbial colonization is a complex process influenced by a two-way interaction between host and microbes and a variety of external factors, including maternal microbiota, the birth process, diet, and antibiotics.

The objective of this study was to evaluate the composition of the total bacteria in the rumen and jejunum of newborn calves, and determine how the composition changes during normal development. Our results suggest that each sampled age group has its distinct microbiota, as reflected by the clustering of the samples by age group, with two different pipelines. 
In this study, the a-diversity of the bacterial community was greatest at the middle-end of the trial, richness was following the adaptation period to the growing diet, and diversity was invariable throughout much of the trial. Bacterial community diversity was lower by the beginning of the trial, increasing in the next weeks following the adaptation period.

Oikonomou et al. (2014) and Klein- Jöbstl et al. (2014) in their studies have described an increase in species richness and diversity with the animals increasing age. Our data agree with Austin et al. (2018) in that the 28-day samples had increased richness compared with samples at weaning, suggesting a stabilization of the microbiome in more mature animals. In contrast, in the study by Klein et al. (2019), richness, diversity, and the number of observed OTUs, decreased significantly between 6 and 24 hours after birth. In the same way, a decrease in mean Chao estimate, Shannon and observed OTUs, during the first day after birth was seen in calves in the studies of Alipour et al. (2018) and Yeoman et al. (2018). Possible explanations given by these authors are selective effects from the environment, especially the diets given to the animals, and a higher diversity caused by prenatal colonization.

The ruminal ecosystem is a complex consortium of different bacterial species living in a symbiotic relationship with the host. Zhou et al. (2015) in their review on rumen bacteria found that in ruminal populations the most abundant phylum always falls on Firmicutes and Bacteroidetes. Similarly, Tapio et al. (2017), report that the main phylum is represented by Firmicutes (55.9-86.8\%) followed by Bacteroidetes (8-24.4\%) and Proteobacteria (0.9-13.4\%), with other phyla such as Actinobacteria, Cyanobacteria representing less than $0.5 \%$.

Next-generation sequencing and bioinformatics tools have greatly advanced our knowledge about the phylogenetic diversity and ecological role of microbes inhabiting the mammalian gut. However, there is a lack of information on the evaluation of these computational tools in the context of the gastrointestinal ruminants microbiome as these programs have mostly been benchmarked on real or simulated datasets generated from human studies. In this study, we also compared the outcomes from two different methods, 1) KRAKEN2, and 2) a pipeline based in USEARCH, to assess the taxonomic profiles (bacteria) of rumen and jejunum microbial communities using DNA sequencing. Our study finds that these programs perform similarly and achieve the same conclusions.

Similarly, Neves et al. (2017), in their study, compared the outcomes of KRAKEN2 and a pipeline developed in-house based on MOTHUR (Schloss 2009; 2020), both approaches revealed a similar phylum distribution of the most abundant taxa, with Bacteroidetes, Firmicutes, and Proteobacteria accounting for approximately $80 \%$ of total bacterial abundance. Similar results were described in the study of Glendinning et al. (2020); they performed a metagenomic analysis of samples taken from the ruminal contents of cattle, sheep, reindeer, and red deer, with the taxonomies assigned with KRAKEN2; in all ruminant species, Bacteroidetes was the most abundant phylum, with Firmicutes being the second. Similarly, Palomba et al. (2017), using USEARCH with rumen and reticulum samples, detected high levels of phylum Planctomycetes; phylum Firmicutes; phylum Bacteroidetes; and phylum Synergistetes), plus 
several other families with a moderate relative abundance (>3\%), belonging to the usual components of the ruminal microbiota of sheep, cattle and dairy cows.

The main identified phylum's and their changes over time in this work were Firmicutes, Proteobacteria, Bacteroidetes, and Actinobacteria. These findings agree with the proportions reported by Jiao et al. (2015), and Malmuthuge et al. (2015), and Sbardellati et al. (2020), using 16S rRNA, observed dominance of Proteobacteria phylum during the first week, but after that were replaced by Firmicutes and Bacteroidetes phyla. Also, O'Hara et al. (2020) in their study using USEARCH commands, found that the proportion of bacteria were manly Firmicutes, Bacteroidetes, and Proteobacteria, also Actinobacteria, Fibrobacteres, Spirochaete, and Cyanobacteria, same as our finding with both programs USEARCH and KRAKEN2.

Gut microbial colonization during the preweaning period has primarily been studied using fecal samples because it is a non-invasive way to collect samples from the same individual over time (Klein et al. 2019; Alipour et al. 2018 and Yeoman et al. 2018). However, Malmuthuge et al. (2014) reported that bacterial composition could vary depending on the GIT region and sample type used (content versus mucosal tissue). Palomba et al. (2017), and Zeng et al. (2017) using USEARCH commands, found an important difference in the taxonomy across the GIT of the pre-weaned lambs' understudy, being Firmicutes and Bacteroidetes the most abundant phylum in all GIT, as in our data wherefrom 7-day the proportion of Firmicutes and Bacteroidetes increase in jejunum; also Taschuk and Griebel (2012) and Klein et al. (2019) reported Firmicutes and Bacteroidetes dominated the bacterial communities along with the GIT and the relative abundance of these 2 phyla varied greatly among local gut regions and between the tissue and content within each region (Malmuthuge and Guan, 2017).

In animals raised on calf starter, GIT microbial communities in those fed silage or mixed diets became more alike and more diverse with age. Animals had a succession of microorganisms representing similar taxa but different specific OTUs present at different ages. Therefore, age is one of the major driving forces in the establishment of microbial communities in developing calves (Jami et al. 2013; DillMcFarland et al. 2017, 2019). Ma et al. (2020) detected changes in gut microbial diversity in healthy calves that were fed only milk replacer (same amount under the same age), and therefore, speculate that host (age and growth) was the main factor driving the development of microbial diversity in the early life of calves. Li et al. (2012), reported that calves fed with a milk substitute and subsequently with a starter feed showed a predominance of the phylum Bacteroidetes during the first 6 weeks of age; this is similar to the results obtained in this work ( $P \leq 0.05$, Fig. $3 c)$.

As we can see throughout many studies, the microbial populations maintain some stability through time, and for that reason, many authors speak of a microbial core. A microbial core is defined as a set of microbial species present in individuals within a given species (Taschuk et al. 2012).

Henderson et al. (2015) carried out a study of the composition of the microbial community of the rumen in different species, including cows, goats, camels, buffaloes, sheep, and how this microbiome could vary by factors like diet and the host, however, thanks to the fact that a central microbiome (a core) is 
hypothesized to be present, variations are small even in a wide geographical range. They collected samples in 35 different countries, where the dominant bacterial phylum was mainly Firmicutes, Bacteroidetes, Proteobacteria, and Fibrobacter (representing about 90\%). The seven most abundant bacterial groups comprised $67.1 \%$ of all bacterial sequence data, were detected in all samples, and can be considered the "dominant" ruminal bacteria. These were Prevotella, Butyrivibrio, and Ruminococcus, as well as Lachnospiraceae, Ruminococcaceae, Bacteroidales, and Clostridiales.

Xue et al. (2018), analyzed the microbiome of 334 dairy cows, finding that there was a bacterial core that represented $46 \%$ of all the bacteria found in the samples, these phyla were Firmicutes $(21.67 \%)$, Bacteroidetes (20.68\%), Proteobacteria (0.52\%).

There is also an intestinal core; Fraune et al. (2007) studied this nucleus over three decades and he defined it as a stable population across samples that is relatively constant, with an endosymbiotic relationship between the host and its microbiota. Taschuk et al. (2012) mention that the central microbiome (core) must be redefined at a functional level, with greater emphasis on the processes carried out by the resident biota, and less emphasis on the absolute or relative abundance of individual organisms.

Therefore, maintaining the tolerance and stability of populations may require the presence and interaction of a relatively consistent and specific microbiota, for example, many diseases, such as inflammatory bowel disease, or ruminal acidosis have been associated with changes in the microbial population, so it is not uncommon to find a species-bacterial core that helps to maintain homeostasis (Fraune et al. 2007; Taschuk et al. 2012).

Research continues to show that small changes in ruminal microbial taxa or the abundance of specific microbes impact livestock productivity. Factors such as age, diet, feeding system, host species, and even geography, can alter the proportions of ruminal microorganisms. Several studies have analyzed different factors that affect the colonization of the digestive tract, such as age, diet, geographic location, feeding system, etc. Most studies concluded that the common factor that affects how populations of the digestive tract microbiota move are diet. We can observe this in works such as those of Malmuthuge et al. (2011), Petri et al. (2013), lqbal et al. (2018), Clemmons et al. (2019), Guo et al. (2020), and many other authors that focus on the relationship between the type of diet or the diet changes and microbiome and how this impacts production.

The microbiota plays a fundamental role in the development of immunity and gastrointestinal metabolism, so knowing these populations allows not only to prevent diseases but also to improve productive parameters. This work used the USEARCH and KRAKEN2 programs, and demonstrated that they both are good tools in the analysis of the GIT microbiota in ruminants; an important point to mention is that we found USEARCH to be user-friendly because it has good technical support, its user manuals remain updated, and they were complete, with explanations of the function and use of each of its commands. Therefore, we recommend USEARCH for those who are beginning in microbiome analysis. Even so, we think that using two pipelines supported by a collection of tools could help control the 
sources of variation present in these kinds of analyses (e.g. analytical pipelines, related databases, and software parameters), which will lead to more reliable biological interpretations and improved taxonomic evaluation at the species level (minimizing unclassified sequences).

Our results showed the profile of microbial colonization of the gastrointestinal tract of 0 to 42 -day-old calves from a tropical region of Mexico. However, it is necessary to analyze other aspects of this colonization and how it can influence factors such as the pattern of microbial succession along with the GIT in pre-ruminant and ruminant animals. This knowledge is essential since it influences the development and maturation of the host's GIT, as well as the development of the immune system and therefore, the health of the animals. In addition, information on the viability, genetic sequences (metagenomics), or even gene expression (metatranscriptomics and metaproteomics) of the described microbial core is required. Therefore, much remains to be understood regarding the underlying mechanisms of possible interactions between the ruminal and intestinal microbial communities and their host. In addition, more emphasis should be placed on dysbiosis caused by antimicrobials in feed and the possibility of using the gut microbiome as prebiotics and probiotics as antimicrobial substitutes. In the future, it will be possible with the collection of microbiota data to create bioinformatics tools, such as algorithms based on the establishment of digestive tract populations to predict patterns with which we can prevent diseases and help design preventive treatment strategies.

\section{Declarations}

Funding This work was supported by PAPIIT-UNAM (Grant number IN211518) to Ofelia Mora.

Conflict of interest The authors declare no competing interests.

Availability of data and material The authors assure that the data and materials support the published claims and comply with field standards. The datasets analyzed during the current study are available from the corresponding author on reasonable request.

Code availability Data were analyzed using SAS (9.3).

Author contributions Ofelia Mora, Armando Shimada, and Carla Daniela Robles-Espinoza contributed to the study conception and design. Material preparation, data collection, and analysis were performed by Carolina Robles and Laura González-Dávalos. The first draft of the manuscript was written by Carolina Robles-Rodríguez and all the authors commented on previous versions of the manuscript. All the authors read and approved the final manuscript.

Ethics approval All the procedures used in this study have been approved by the internal committee for the experimental animal care and use (CICUAE.DC-2019/4-2, UNAM) according to the NOM-062-ZOO1999.

Consent to participate Not applicable. 
Consent for publication Not applicable

Acknowledgments We would like to thank Luis Aguilar, Alejandro de León, Carlos Flores, and Jair García from Laboratorio Nacional de Visualización Científica Avanzada-UNAM; Miriam Bravo López and Carolina Rocha from Laboratorio Internacional de Investigación sobre el Genoma Humano (LIIGH)-UNAM; and Mariana Isabel Olivares Salazar from CEIEGT-UNAM for their technical support. Carolina RoblesRodríguez thanks CONACYT for scholarship at PCiPSA-UNAM.

\section{References}

1. Alipour, M., Jalanka, J., Pessa-Morikawa, T., Kokkonen, T., Satokari, R., Hynonen, U., 2018. The composition of the perinatal intestinal microbiota in cattle. Scientific Reports, 8, 10437.

2. Austin, K. J., Cunningham, H. C., Powell, S. R., Carpenter, K. T., Cammack, K. M., 2018. Investigation of maternal breed and rearing type on the calf rumen microbiome from day 28 through weaning. Translational Animal Science, 2, 125-129.

3. Bolger, A. M., Lohse, M., Usadel, B., 2014. Trimmomatic: a flexible trimmer for Illumina sequence data. Bioinformatics 30, 2114.

4. Clemmons, B., Martino, C., Schneider, G., Lefler, J., Embree, M., Myer, R., 2019. Temporal stability of the ruminal bacterial communities in beef steers. Scientific Reports, 9, 9522.

5. Dill-McFarland, K., Breaker, J., Suen, G., 2017. Microbial succession in the gastrointestinal tract of dairy cows from 2 weeks to the first lactation. Scientific Reports, 7, 40864.

6. Dill-McFarland, K., Weimer, J., Breaker, J., Suen, G., 2019. Diet influences early microbiota development in dairy calves without long-term impacts on milk production. Applied and Environmental Microbiology, 85, e02141-18.

7. Edgar, R., 2010. Search and clustering orders of magnitude faster than BLAST. Bioinformatics, 26, 2460-2461.

8. Fraune S., Bosch T., 2007. Long-term maintenance of species-specific bacterial microbiota in the basal metazoan Hydra. Proceedings of the National Academy of Sciences of the United States of America 104, 13146-13151.

9. Guo, J., Li, P., Liu, S., Miao, B., Zeng, B., Jiang, Y., Zhang, H., 2020. Characterization of the rumen microbiota and volatile fatty acid profiles of weaned goat kids under shrub-grassland grazing and indoor feeding. Animals (Basel), 10, 176.

10. Henderson, G., Cox, F., Ganesh, S., Jonker, A., Young, W., Abecia, L., Attwood, G.T., 2015. Rumen microbial community composition varies with diet and host, but a core microbiome is found across a wide geographical range. Scientific Reports, 5, 14567.

11. Iqbal, M, Zhang, Q., Yang, Y., Zou, C., Li, L., Liang, X., Lin, B., 2018. Ruminal fermentation and microbial community are differently influenced by four typical subtropical forages in vitro. Animal Nutrition, 4, 100. 
12. Jami, E., Israel, A., Kotser, A., Mizrahi, I., 2013. Exploring the bovine rumen bacterial community from birth to adulthood. The ISME Journal, 7, 1069-1079.

13. Jiao, J., Huang, J., Zhou, C., Tan, Z., 2015. Taxonomic identification of ruminal epithelial bacterial diversity during rumen development in goats. Applied and Environmental Microbiology, 81, 3502.

14. Klein, D., Quijada, M., Dzieciol, M., Feldbacher, B., Wagner, M., Drillich, M., Mann, E., 2019. Microbiota of newborn calves and their mothers reveals possible transfer routes for newborn calves' gastrointestinal microbiota. PloS ONE, 14, e0220554.

15. Klein-Jöbstl, D., Schornsteiner, E., Mann, E., Wagner, M., Drillich, M., Schmitz-Esser, S., 2014. Pyrosequencing reveals diverse fecal microbiota in Simmental calves during early development. Frontiers in Microbiology, 5, 622.

16. Li, W., Connor, E., Baldwin, L., Sparks, L., 2012. Characterization of the rumen microbiota of preruminant calves using metagenomic tools. Environmental Microbiology, 14, 129-139.

17. Liang, G., Malmuthuge, N., Bao, H., Stothard, P., Griebel, J., 2016. Transcriptome analysis reveals regional and temporal differences in mucosal immune system development in the small intestine of neonatal calves. BMC Genomics, 17, 602.

18. Liang, G., Malmuthuge, N., McFadden, T., Bao, H., Griebel, J., Stothard, P., 2014. Potential regulatory role of microRNAs in the development of bovine gastrointestinal tract during early life. PloS ONE, 9, e92592.

19. Lu, J., Salzberg, S. L., 2020. Ultrafast and accurate 16S rRNA microbial community analysis using Kraken 2. Microbiome, 8, 1.

20. Ma, T., Villot, C., Renaud, D., Skidmore, A., Chevaux, E., Steele, M., 2020. Linking perturbations to temporal changes in diversity, stability, and compositions of neonatal calf gut microbiota: prediction of diarrhea. The ISME Journal, 14, 2223-22353.

21. Malmuthuge, N., Li, M., Chen, Y., Fries, P.N., Griebel, P.J., Baurhoo, B., Zhao, X., Guan, L., 2011. Distinct commensal bacteria associated with ingesta and mucosal epithelium in the gastrointestinal tracts of calves and chickens. FEMS Microbiology Ecology, 79, 337-347.

22. Malmuthuge, N., Liang, G., Griebel, J., 2014. Taxonomic and functional compositions of the small intestinal microbiome in neonatal calves provide a framework for understanding early life gut health. Applied and Environmental Microbiology, 85, e02534-e02538.

23. Malmuthuge, N., Griebel, J., Guan, L., 2015. The gut microbiome and its potential role in the development and function of newborn calf gastrointestinal tract. Frontiers in Veterinary Science, 2, 36.

24. Malmuthuge, N., Guan, L., 2017. Understanding the gut microbiome of dairy calves: opportunities to improve early-life gut health. Journal of Dairy Science, 100, 5996-6005.

25. McMurdie, P.J., Holmes, S., 2013. Phyloseq: an R package for reproducible interactive analysis and graphics of microbiome census data. PloS ONE, 8, e61217.

26. Neves, L., Li, F., Ghoshal, B., McAllister, T., Guan, L., 2017. Enhancing the resolution of rumen microbial classification from metatranscriptomic data using Kraken and Mothur. Frontiers in 
Microbiology, 8, 2445.

27. O'Hara, E., Neves, A. L., Song, Y., \& Guan, L. L., 2020. The Role of the Gut Microbiome in Cattle Production and Health: Driver or Passenger? Annual review of animal biosciences, 8, 199-220.

28. Oikonomou, G., Teixeira, A.G.V., Foditsch, C., Bicalho, M.L., Machado, V.S., Bicalho, R.C., 2013. Fecal microbial diversity in pre-weaned dairy calves as described by pyrosequencing of metagenomic 16S rDNA. Associations of Faecalibacterium species with health and growth. PloS ONE, 8, e63157.

29. Palomba, A., Tanca, A., Fraumene, C., Abbondio, M., Fancello, F., Atzori, A.S., Uzzau, S., 2017. Multiomic biogeography of the gastrointestinal microbiota of a pre-weaned lamb. Proteomes, 5, 36.

30. Petri, M., Schwaiger, T., Penner, B., Beauchemin, A., Forster, R. J., McKinnon, J., McAllister, A., 2013. Characterization of the core rumen microbiome in cattle during the transition from forage to concentrate as well as during and after an acidotic challenge. PloS ONE, 8, e83424.

31. Pitta, D.W., Pinchak, W.E., Dowd, S.E., Osterstock, J., Gontcharova, V., Youn, E., Wickersham, T.A., 2010. Rumen bacterial diversity dynamics associated with changing from Bermudagrass hay to grazed winter wheat diets. Microbial Ecology, 59, 511-522.

32. Sbardellati, L., Fischer, A., Cox, S., Li, W., Kalscheur, F., Suen, G., 2020. The bovine epimural microbiota displays compositional and structural heterogeneity across different ruminal locations. Journal of Dairy Science, 103, 3636-3647.

33. Schloss, P.D, Westcott, S.L., Ryabin, T., Hall, J.R., Hartmann, M., Hollister, E.B., Lesniewski, R.A., Oakley, B.B., Parks, D.H., Robinson, C.J., Sahl, J.W., Stres, B., Thallinger, G.G., Van Horn, D.J., Weber, C.F., 2009. Introducing mothur: open-source, platform-independent, community-supported software for describing and comparing microbial communities. Applied and Environmental Microbiology, 75, 7537-7541.

34. Schloss, P.D., 2020. Reintroducing mothur: 10 years later. Applied and Environmental Microbiology, 86, e02343-19.

35. Tapio, I., Fischer, D., Blasco, L., Tapio, M., Wallace, R. J., Bayat, A., Vilkki, J., 2017. Taxon abundance, diversity, co-occurrence and network analysis of the ruminal microbiota in response to dietary changes in dairy cows. PloS ONE, 12, e0180260.

36. Taschuk, R., Griebel, P., 2012. Commensal microbiome effects on mucosal immune system development in the ruminant gastrointestinal tract. Animal Health Research Reviews, 13, 129-141.

37. Wood, D.E., Lu, J., Langmead, B., 2019. Improved metagenomic analysis with Kraken 2. Genome Biology, 20, 257.

38. Xue, M., Sun, H., Wu, X., Liu, J., 2018. Assessment of rumen microbiota from a large dairy cattle cohort reveals the pan and core bacteriomes contributing to varied phenotypes. Applied and Environmental Microbiology, 84, e00970-18.

39. Yeoman, J., Ishaq, S.L., Bichi, E., Olivo, S.K., Lowe, J., Aldridge, B.M., 2018. Biogeographical differences in the influence of maternal microbial sources on the early successional development of the bovine neonatal gastrointestinal tract. Scientific Reports, 8, 3197. 
40. Yu, Z., and Morrison, M., 2004. Improved extraction of PCR-quality community DNA from digesta and fecal samples. BioTechniques, 36, 808-812.

41. Zeng, Y., Zeng, D., Ni, X., Zhu, H., Jian, P., Zhou, Y., Jing, B., 2017. Microbial community compositions in the gastrointestinal tract of Chinese Mongolian sheep using Illumina MiSeq sequencing revealed high microbial diversity. AMB Express, 7, 75.

42. Zhong, T., Hu, J., Xiao, P., Zhan, S., Wang, L., Guo, J., Li, L., Zhang, H., Niu, L., 2017. Identification and characterization of microRNAs in the goat (Capra hircus) rumen during embryonic development. Frontiers in Genetics, 8, 163

43. Zhou, M., Chen, Y. Guan, L., 2015. Rumen Bacteria. In: A. Puniya, R. Singh, D. Kamra (eds), Rumen Microbiology: From Evolution to Revolution, Springer, New Delhi, 79-95.

\section{Figures}



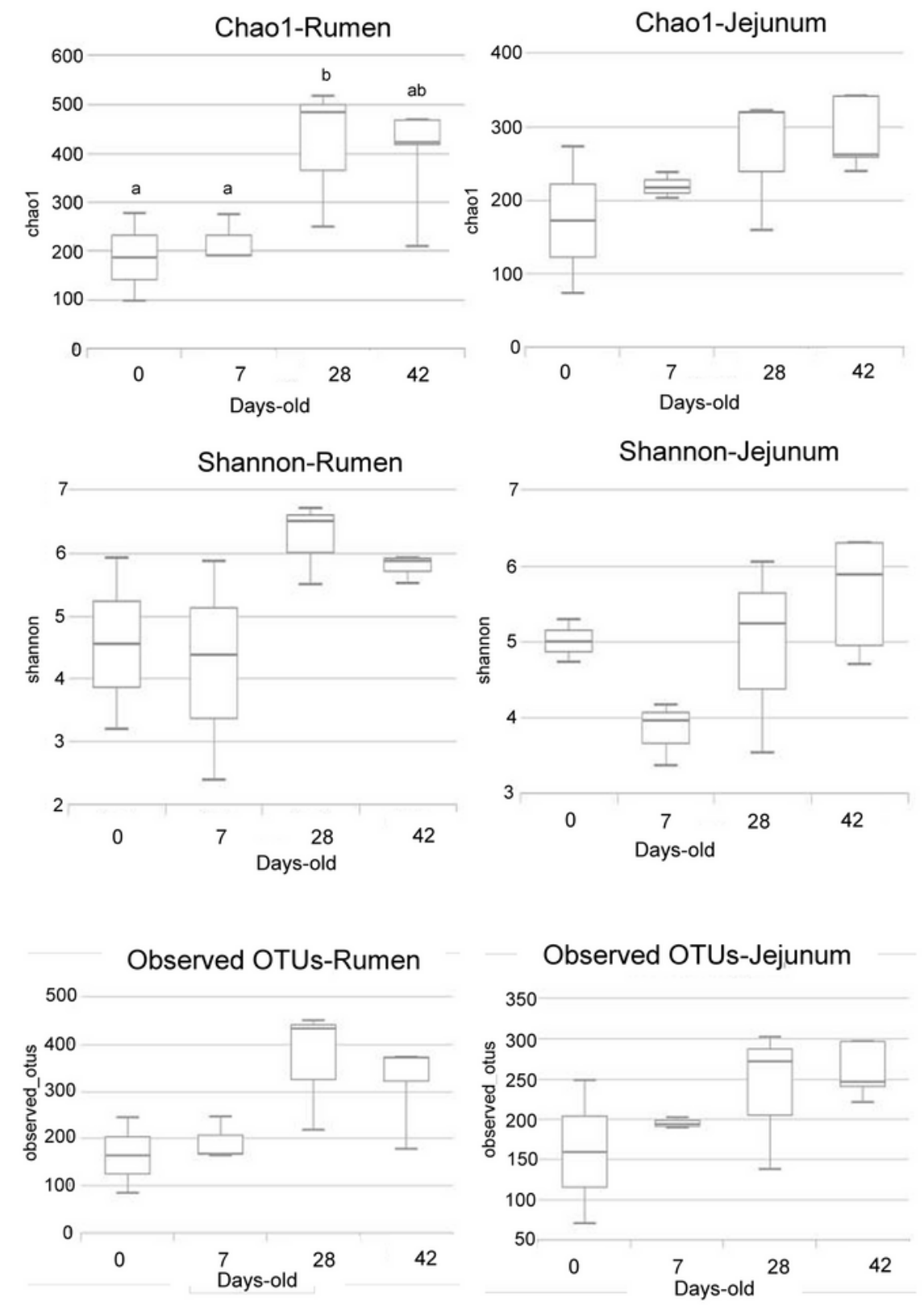

\section{Figure 1}

Alpha diversity. Temporal changes of the rumen and jejunum bacterial diversity, richness, and observed otus in calves $0,7,28,42$ days old from the Mexican tropics. Data are visualized as box-and-whisker plots showing the median and the interquartile (midspread) range (boxes containing $50 \%$ of all values), the whiskers (representing the 25 and 75 percentiles), and the extreme data points. Letters above boxes indicate significant differences $(n=3, P \leq 0.05)$ 


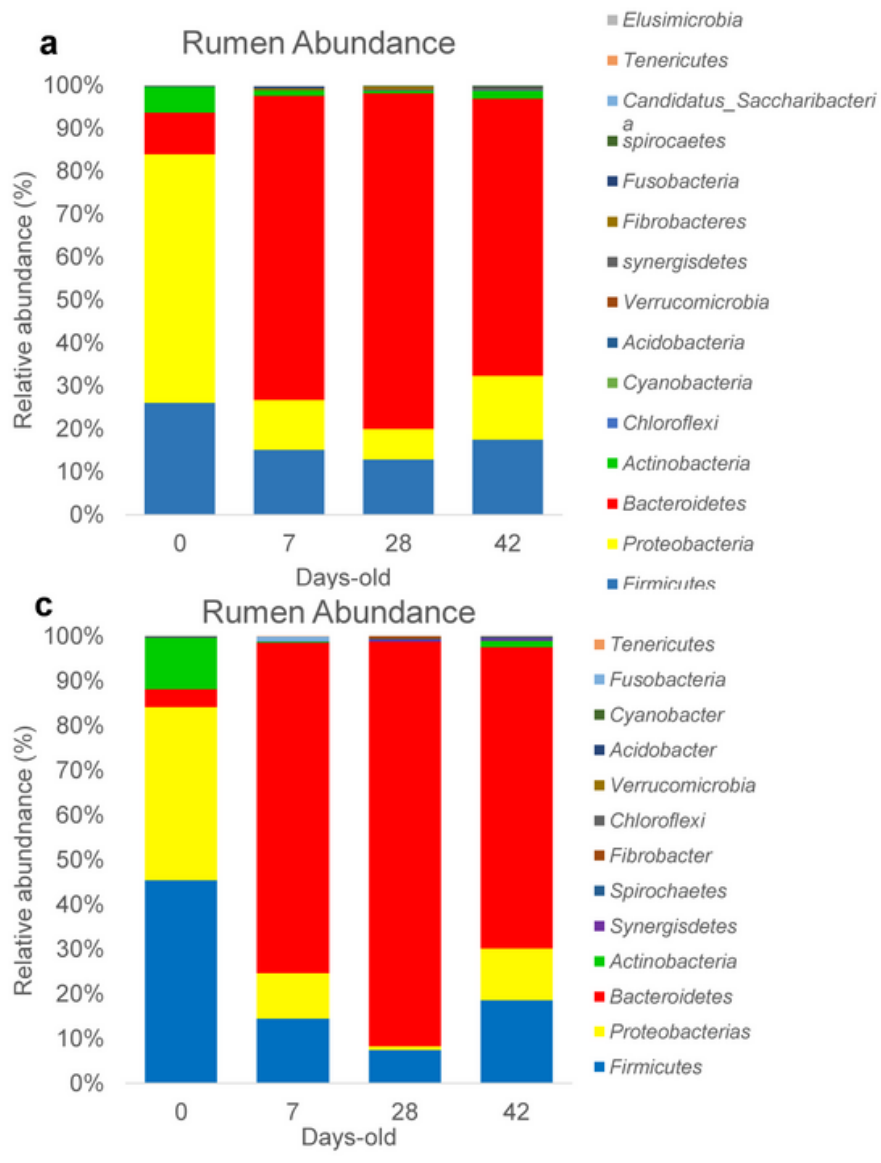

b
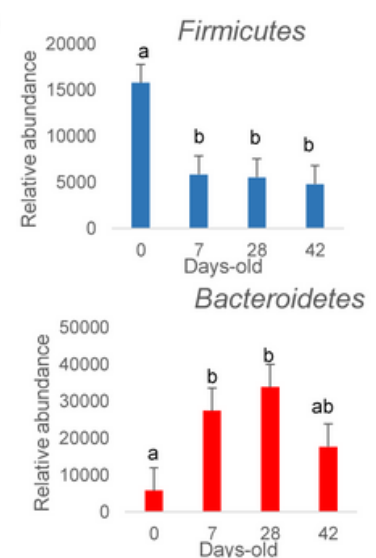

d
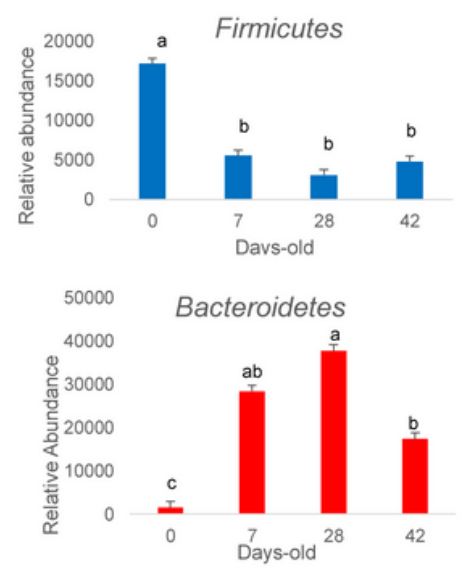
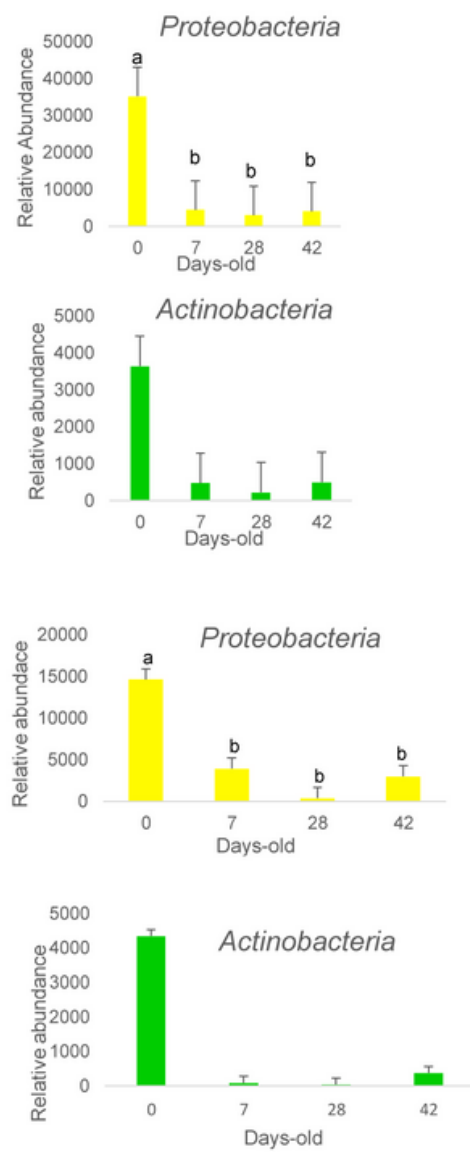

Figure 2

Temporal variations in the rumen. a) Relative abundance of rumen phyla in calves $0,7,28,42$ days old from the Mexican tropics (detected by USEARCH). b) Changes over time of the relative abundance of the most representative phyla that inhabit the rumen (detected by USEARCH) c) Relative abundance of rumen phyla in calves $0,7,28,42$ days old from the Mexican tropics (detected by KRAKEN2). d) Changes over time of the relative abundance of the most representative phyla that inhabit the rumen (detected by KRAKEN2). Different literals between columns indicate statistical difference $(n=3, P \leq 0.05)$ 

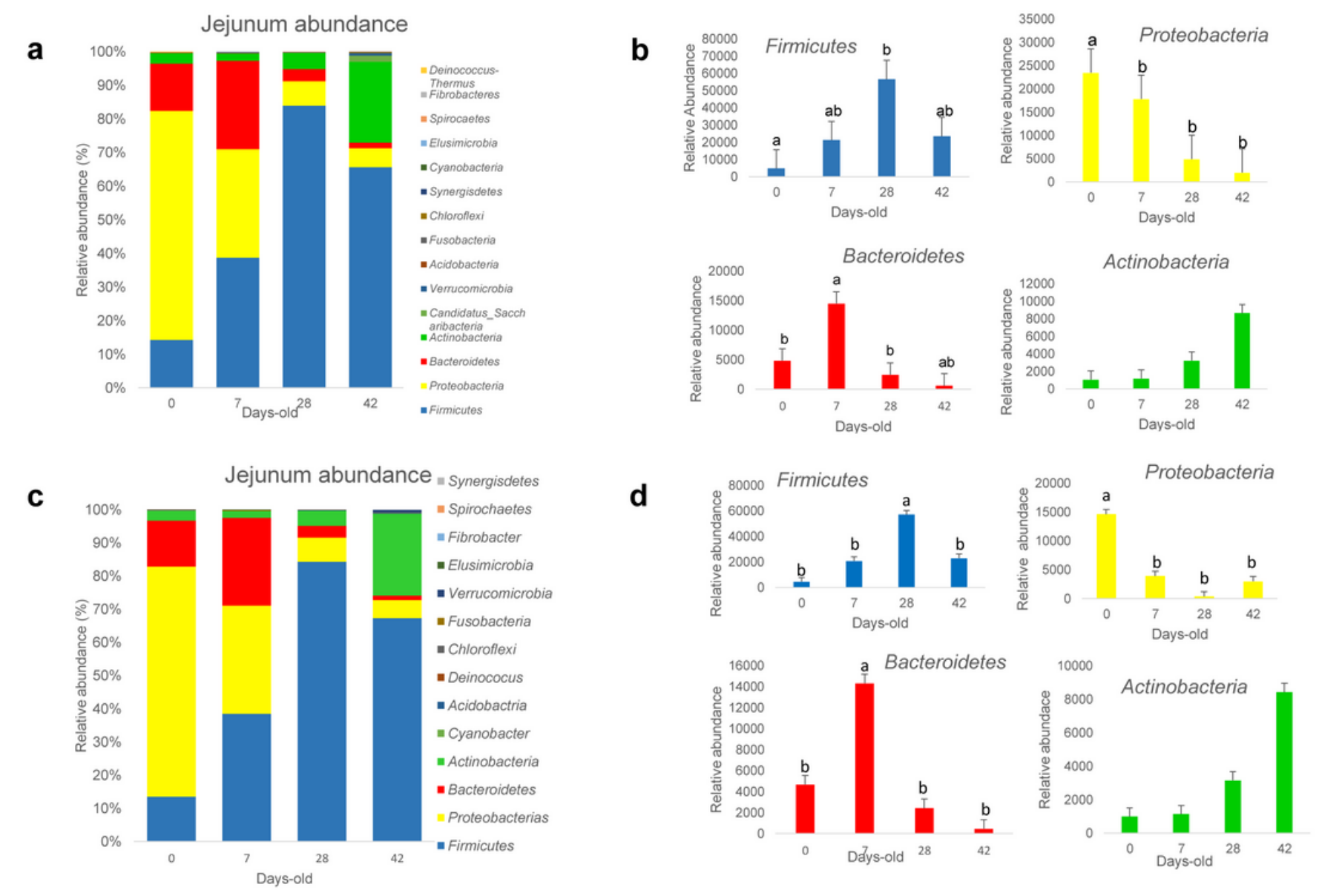

\section{Figure 3}

Temporal variations in the jejunum. a) Relative abundance of jejunum phyla in calves $0,7,28,42$ days old from the Mexican tropics (detected by USEARCH). b) Changes over time of the relative abundance of the most representative phyla that inhabit the jejunum (detected by USEARCH). c) Relative abundance of jejunum phyla in calves $0,7,28,42$ days old from the Mexican tropics (detected by KRAKEN2). d) Changes over time of the relative abundance of the most representative phyla that inhabit the jejunum (detected by KRAKEN2). Different literals between columns indicate statistical difference $(n=3, P \leq 0.05)$ 

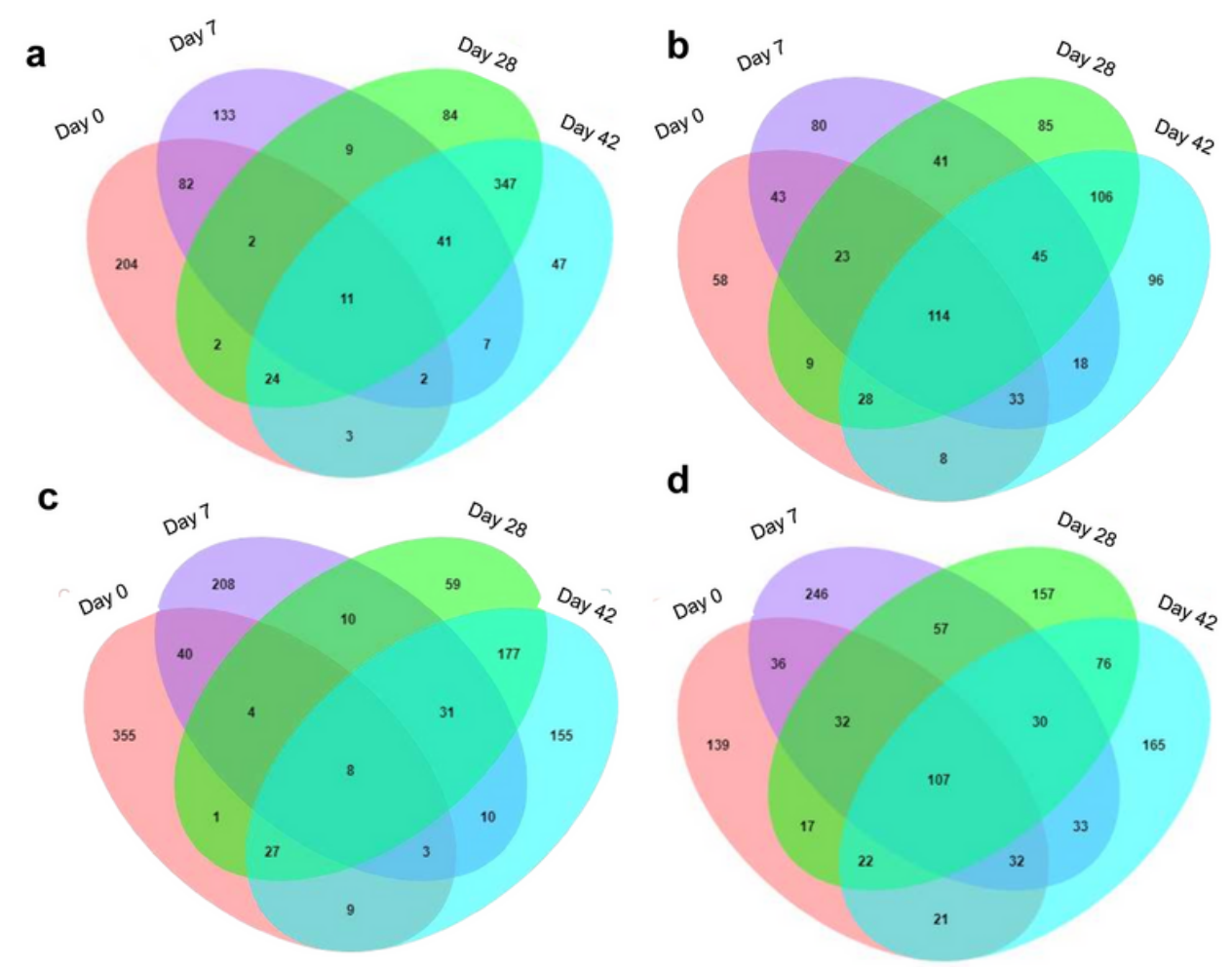

\section{Figure 4}

Shared OTUs across different groups. Venn plot showing the shared and unique OTUs found in each plotted group. a) Shared OTUs across rumen sample (USEARCH). b) Shared OTUs across the jejunum sample (USEARCH). c) Shared OTUs across rumen sample (KRAKEN2). d) Shared OTUs across jejunum sample (KRAKEN2) 